\title{
Identification of Natural Bamboo Fiber and Regenerated Bamboo Fiber by the Method of Modified near Infrared Spectroscopy
}

\author{
Weidong $\mathrm{Li}^{1}$, Zhixiong $\mathrm{Li}^{1}$ and Xinhou Wang ${ }^{2}$ \\ ${ }^{1}$ Shanghai Institute of Quality Inspection and Technical Research, Shanghai 200040, P. R. China \\ ${ }^{2}$ Donghua University, Shanghai 201620, P. R. China
}

\begin{abstract}
In this work, the applicability and effectiveness of identification of natural bamboo fiber and regenerated bamboo fiber by near infrared spectroscopy are investigated. A discrimination model based on Ward's algorithm and Hierarchical Cluster Analysis is pretreated by the first derivative and vector normalization, which can be used to distinguish natural bamboo fiber and regenerated bamboo fiber. In addition, the near infrared spectra model exhibits a high accuracy with an effective identification of the double fibers.
\end{abstract}

\section{Introduction}

Bamboo fiber, as a kind of renewable, biodegradable, low-priced and abundant cellulosic fibers, it has been widely used in textiles and clothing by virtue of their excellent performance of physics and chemistry [1-4]. Bamboo fiber consists of natural bamboo fiber and regenerated bamboo fiber [5]. Natural bamboo fiber is fabricated by removing plant tissues without cellulose based on the mechanical and physical methods [6]. The fabrication of regenerated bamboo fiber is similar to the common viscose fiber, which is prepared by chemical spinning solution [1].

The structure and function is different between natural bamboo fiber and regenerated bamboo fiber due to their different manufacturing process. At present, some methods and papers on the identification of bamboo fibers have been put forward, but their applicability and effectiveness are unsatisfactory [7-9]. In this paper, natural bamboo fibers and regenerated bamboo fiber produced by the main domestic manufacturers are collected widely, and the accurate identification of these two kinds of fibers is realized by the near infrared spectroscopy (NIR) technology in virtue of the cluster analysis model.

\section{Experimental section}

\subsection{Materials}

The materials used in the experiments are listed in Table 1. Natural bamboo fibers supplied by Sichuan Banbo
Bamboo Development Co., Ltd and Fujian Zhenghe Bambootextile Co., Ltd; bamboo pulp viscose fibers supplied by Jigao Chemical Fiber Co., Ltd of Hebei provinceand Suzhou Shenboo Textile Co., Ltd.

Table 1. Materials used for the NIR spectra analysis

\begin{tabular}{ccc}
\hline Fiber samples & $\begin{array}{c}\text { Number of the } \\
\text { modeling } \\
\text { sample set }\end{array}$ & $\begin{array}{c}\text { Number of the } \\
\text { correction } \\
\text { sample set }\end{array}$ \\
\hline $\begin{array}{c}\text { Natural Bamboo } \\
\text { fibers }\end{array}$ & 15 & 4 \\
$\begin{array}{c}\text { Regenerated bamboo } \\
\text { fiber }\end{array}$ & 10 & 4 \\
\hline
\end{tabular}

(Note: fibers used for the NIR analysis are numbered in random order)

\subsection{Instruments}

MPA multi-functional Fourier transform near infrared spectrometer (Bruker Co.). Diffuse reflectance integrating sphere attachment (built-in gold-plated background), gold-plated integrating sphere diameter is $10 \mathrm{~cm}$, measurement window diameter is $2 \mathrm{~cm}, \mathrm{PbS}$ detector. Scanning resolution is $4 \mathrm{~cm}^{-1}$, the number of scans is 32 , spectral range $12000-4000 \mathrm{~cm}^{-1}$, the OPUS software.

\section{Results and discussion}

The NIR spectra of the natural bamboo fiber and regenerated bamboo fiber measured by diffuse reflection method are shown in Figure 1A and 1B, respectively. 

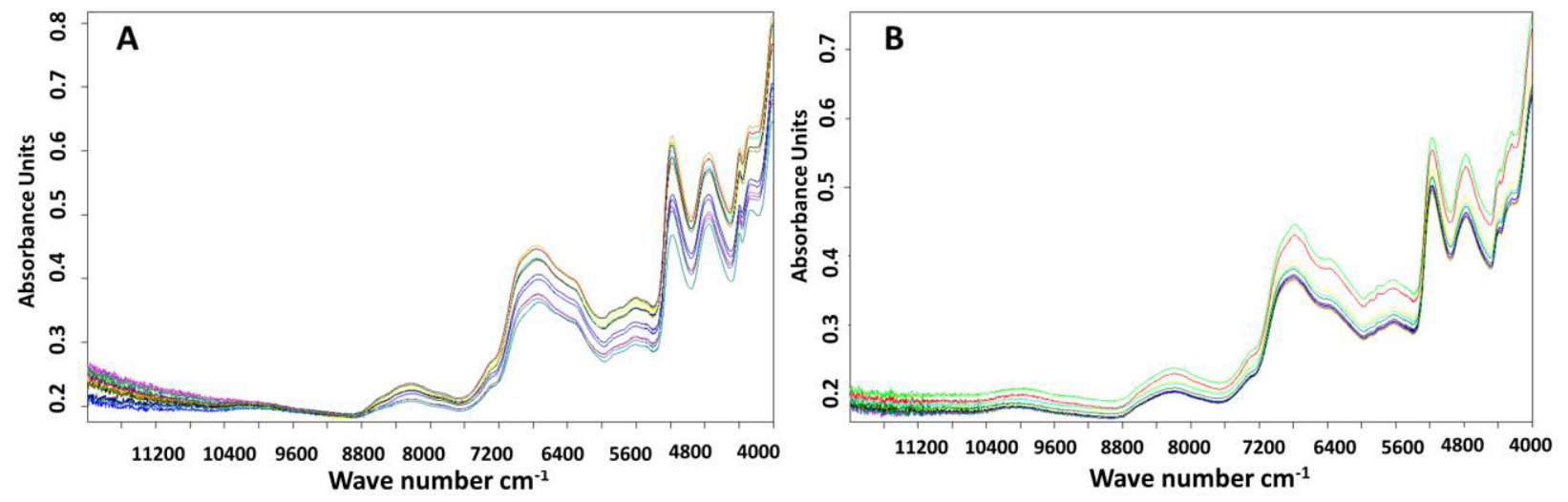

Figure 1. NIR spectra of (A) natural bamboo fiber and (B) regenerated bamboo fiber.

The NIR spectra of natural bamboo fiber and regenerated bamboo fiber are similar, the obvious absorption peaks at $4000 \sim 8800 \mathrm{~cm}^{-1}$ can be observed. Therefore, the first derivative is proposed to eliminate the influence of baseline and background with 10 smooth points, which can intensify the character of respective NIR spectra and keep the effective information. Vector normalization is adopted to eliminate the interference caused by the locations, thickness, manufacturers and producing areas guaranteeing a reliable NIR spectra information.

Then, Ward's algorithm model is employed to calculate the distance based on a view of variance analysis and heterogeneous definition rule. As an excellent methodology to distance's measure, Ward's algorithm model displays an optimized result with a smaller congeneric variance and a larger heterogeneous variance.

Finally, a discrimination model based on Ward's algorithm and Hierarchical Cluster Analysis is built with the help of optimized NIR spectra [10]. As a result, the identification of natural bamboo fiber and regenerated bamboo fiber is realized (Figure 2).

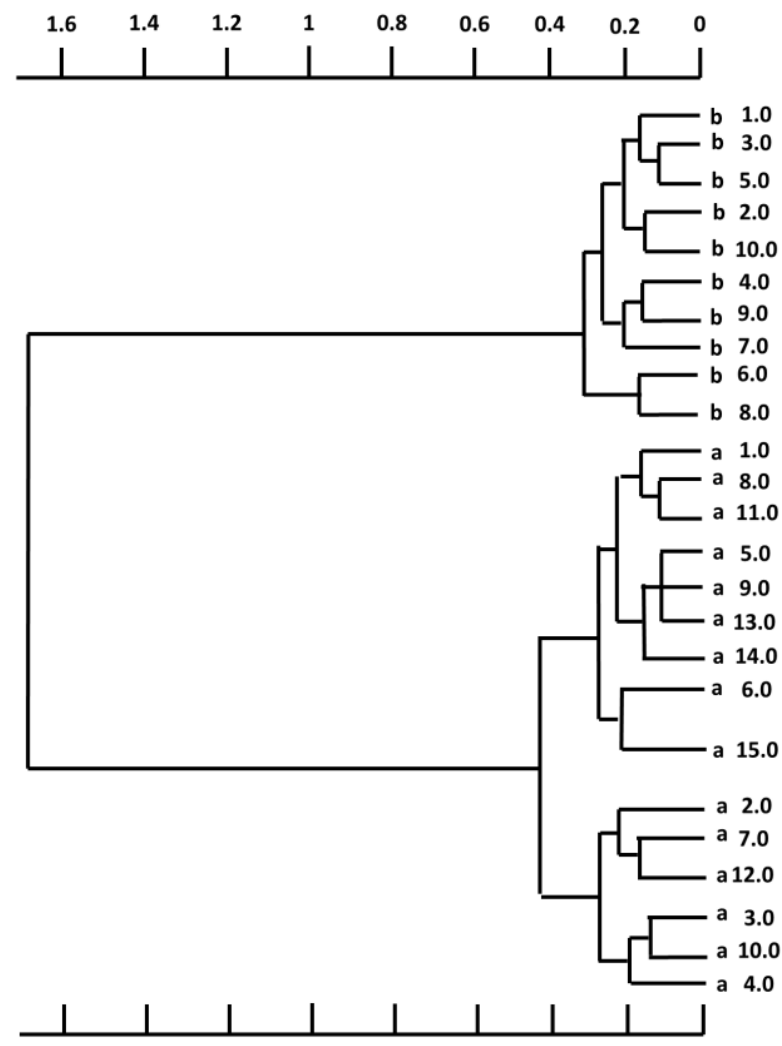

Figure 2. Cluster analysis model of natural bamboo fibers and regenerated bamboo fiber. (Note: a represents natural bamboo fiber, $b$ represents regenerated bamboo fiber. The coordinate value represents the value of the distance between groups)

An accuracy and effective verification of the discrimination model is necessary. The NIR spectra of fiber sample which is pretreated by the first derivative and vector normalization is shown in Figure 3. The verified analysis model and overall verified results are displayed in Figure 3 and Table 2, respectively. 


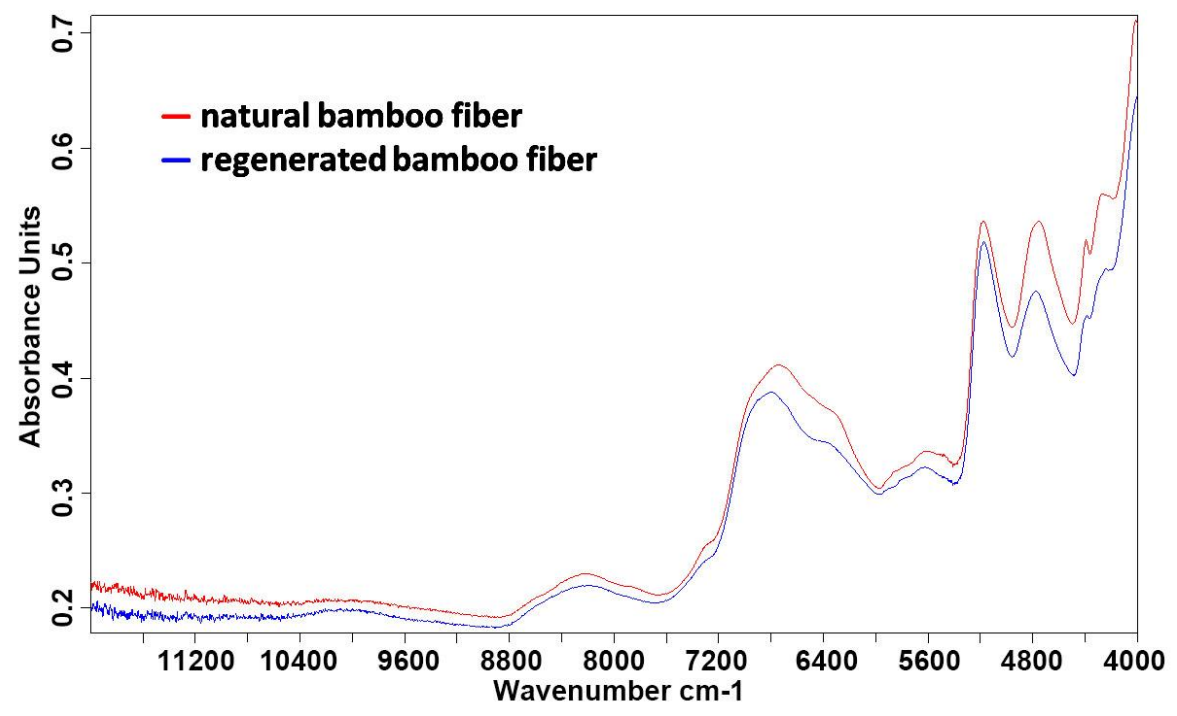

Figure 3. Correctional NIR spectra of the fiber sample set (part for example).
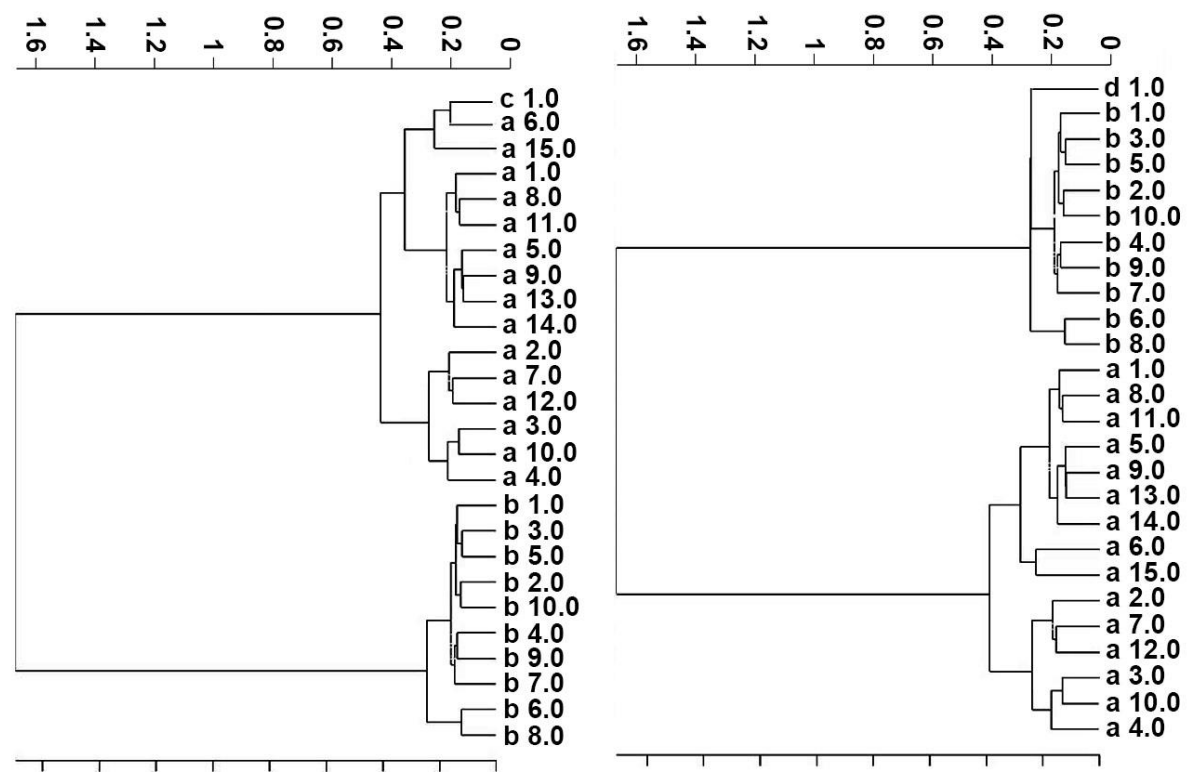

Figure 4. Verified results of cluster analysis model on natural bamboo fiber and regenerated bamboo fiber. (Note: a represents natural bamboo fiber, $b$ represents natural bamboo fibers, $\mathrm{c}$ represents natural bamboo fiber of correction sample set, $d$ represents regenerated bamboo fiber of correction sample set. The coordinate value represents the value of the distance between groups)

Table 2. Overall verified results due to NIR spectra and cluster analysis model

\begin{tabular}{ccccc}
\hline Samples & $\begin{array}{c}\text { Number of the correction } \\
\text { sample set }\end{array}$ & Right results & Wrong results & Model accuracy (\%) \\
\hline $\begin{array}{c}\text { natural bamboo fibers } \\
\text { regenerated bamboo } \\
\text { fiber }\end{array}$ & 4 & 4 & 0 & 100 \\
\hline
\end{tabular}

The identification of the two fibers can be realized with relatively high accuracy based on the results in figure 4 and table 2. In addition, the cluster analysis model established by Hierarchical Cluster Analysis can identify natural bamboo fiber and regenerated bamboo fiber simply and effectively without any damage to the fiber samples. And in this system, the accuracy increases with the growing of representativeness and class of fiber samples, which shows a great potential in the field of sensors and detection.

\section{Conclusion}

In a word, a discrimination model based on Ward's algorithm and Hierarchical Cluster Analysis is pretreated by the first derivative and vector normalization, which achieve the effective identification of natural bamboo fiber and regenerated bamboo fiber with an excellent operability, veracity and detection speed. The method of modified near infrared spectroscopy can be widely used in the field of the identification between similar natural 
and regenerated fibers, showing an extensive application for the sensor, identification and detection.

\section{References}

1. S. Y. Sui and R. Q. Li, A study of structure and performance of bamboo fibers, Journal of Textile Research, 24 (2016), 535-536.

2. S. Y. Lee, S. J. Chun, G. Doh, et al., Influence of chemical modification and filler loading on fundamental properties of bamboo fibers reinforced polypropylene composites, Journal of Composite Materials, 43 (2009), 1639-1657.

3. S. B. Ma and P. Y. Wu, Identification and performance analysis of bamboo pulp fiber and viscose fiber, Wool Textile Journal, 38 (2010), 42-46.

4. T. Zhang, W. B. Bao and J. Y. Yu, Study on identification of bamboo fiber, Journal of Textile Research, 25 (2004), 28-29.
5. W. D. Li, Research progress in determination methods of bamboo fiber, China Fiber Inspection, 359 (2010), 61-64.

6. L. D. Cheng, X. L. Xu and J. H. Lao, An analysis on the morphological structure and property of bamboo fiber, China Textile Leader, 5 (2003), 101-103.

7. Q. B. Zhou and J. L. Chen, The performance difference between bamboo pulp fibre and bamboo fiber, Advanced Textile Technology, 2 (2007), 1-3.

8. J. P. Zhou, Study on the structure and property of original bamboo fiber and bamboo pulp fiber, Shanghai Textile Science \& Technology, 34 (2006), 59-60.

9. J. J. Sun, J. Y. Tian and Z. Y. Gu, Comparison of structure and thermal property between bamboo fibers and regenerated bamboo fibers, Journal of Tianjin Polytechnic University, 25 (2006), 37-40.

10. J. X. Liu, Practical technology of the near infrared spectra analysis, Beijing: Science Press, 2007. 